\title{
The Big Plant Theory y otras metodologías dinamizadoras en grupos reducidos de dos materias de Fisiología Vegetal del grado en Biología
}

\author{
Díaz Varela, José; Carrillo Barral, Néstor; Bernal Pita da Veiga, María de los Ángeles
}

Departamento de Bioloxía, Facultade de Ciencias

\section{RESUMEN}

En este trabajo se describen dos experiencias basadas en la aplicación de estrategias motivadoras a los grupos reducidos de dos materias troncales del Grado en Biología. Ambas son materias del área de Fisiología Vegetal, pero con diferentes características y contextos, por lo que se han usado metodologías diferentes. Una es una materia de segundo curso (Fisiología Vegetal II) con muchos estudiantes por grupo y pocas horas de grupo reducido y otra de tercer curso (Fisiología Vegetal Aplicada) con más horas y menos estudiantes. En Fisiología Vegetal II se han desarrollado dos juegos de preguntas y respuestas y en Fisiología Vegetal Aplicada diferentes actividades (juegos de rol, debates, vídeos, congresos, etc.). Los resultados en ambos casos han sido positivos tanto en motivación como en desarrollo de un pensamiento crítico, y han reforzado entre los estudiantes el valor del trabajo en equipo y de los conocimientos del campo de la Fisiología Vegetal.

PALABRAS CLAVE: Grupos reducidos, Fisiología Vegetal, motivación, participación 


\section{CITA RECOMENDADA:}

Díaz Varela, J., Carrillo Barral, N., Bernal Pita da Veiga, M.A.(2018). The Big Plant Theory y otras metodologías dinamizadoras en grupos reducidos de dos materias de Fisiología Vegetal del grado en Biología. En E. de la Torre Fernández, (ed.) (2018). Contextos universitarios transformadores: retos e ideas innovadoras. II Xornadas de Innovación Docente. Cufie. Universidade da Coruña. (pág. 377-388). DOI capítulo: https://doi.org/10.17979/spudc.9788497496780.377

DOI libro: https://doi.org/10.17979/spudc. 9788497496780

\section{ABSTRACT}

In this paper we describe two experiences based on the application of strategies that motivate students. The experiences correspond to the seminar sessions of two core subjects of the degree in Biology. Both subjects belong to the field of Plant Physiology, but they have different features and context. Therefore, the methodology was different. The first subject (Plant Physiology II) is taught in the second course of Biology, and there are many enrolled students and few hours for seminars. The other subject (Applied Plant Physiology) is taught in the third course, and there are less students and more hours for seminars. In Plant Physiology II the students have to develop and play a game of questions and answers, whereas in Applied Plant Physiology the activities are more varied (role-playing, debate, video, scientific conferences, etc.). In both cases, the results were successful, and the activities motivate the students and contribute to the acquisition of critical thinking. Moreover, they convinced the students of the value of both the teamwork and the knowledge in the field of Plant Physiology.

KEY WORDS: Seminars, Plant Physiology, motivation, participation 


\section{INTRODUCCIÓN}

Tenemos la suerte de decir que nos apasiona nuestro trabajo. Antes de la implantación del Plan Bolonia en 1999, nuestro tipo de docencia estaba eminentemente centrado en la docencia mediante clases magistrales. Este tipo de aprendizaje se caracteriza por un papel pasivo del estudiante en dónde el profesor imparte su conocimiento de una manera activa. Debo de decir que para nosotros era y es una experiencia altamente satisfactoria.

La llegada del Plan Bolonia supuso un cambio de paradigma a nivel educativo con importantes reformas en el seno de la docencia universitaria, tanto a nivel de diseño curricular como de metodologías docentes, y los estudios de Biología no son una excepción (Susanne, 2003). De un tipo de docencia basada exclusivamente en las clases magistrales y en las clases prácticas de laboratorio para las ramas experimentales, se pasó a un tipo de docencia caracterizada por la evaluación continua, los estudios prácticos, la incorporación de las TICs, el uso de las redes sociales, la tutorización individual y el trabajo en grupo. Una de las novedades más importantes en la implantación del Plan Bolonia era la docencia en grupos reducidos, entendiéndolo como una modalidad de trabajo más que un número determinado de estudiantes. Este tipo de docencia implica por parte del estudiante una participación activa en dónde el trabajo autónomo y continuado del estudiante es una parte fundamental del proceso de aprendizaje.

La experiencia que se presenta corresponde a los grupos reducidos de dos materias troncales del grado en Biología de la Universidade da Coruña, que se imparten en segundo (Fisiología Vegetal II) y tercer curso (Fisiología Vegetal Aplicada). En ambos casos el enfoque didáctico está dirigido a motivar a los estudiantes para que se impliquen en el proceso de aprendizaje y para que tengan una actitud crítica. No obstante, al tratarse de materias diferentes, la base de los estudiantes y la organización también es distinta. 


\section{DESCRIPCIÓN DE LA EXPERIENCIA}

\subsection{The Big Plant Theory \& Sayonara Baby}

En el Grado de Biología, la Fisiología Vegetal II es una materia troncal de $2^{\circ}$ de Grado con 6 créditos ECTS que se reparten en clases magistrales, clases prácticas de laboratorio y docencia en grupos reducidos. Se imparte en el segundo cuatrimestre. En el curso 2016.2017 el número de estudiantes matriculados fue de 133 estudiantes, que se repartían en 8 grupos reducidos con una composición de entre 11 y 19 estudiantes por grupo.

En esta materia de segundo se dispone de dos sesiones de dos horas (cuatro horas en total) y los estudiantes aún no tienen fijados los conocimientos básicos propios de la Fisiología Vegetal, por tanto, se trata de reforzar conceptos y apreciar su utilidad. Para ello, y para involucrarlos en el aprendizaje se les plantearon dos juegos, que denominamos "The Big Plant Theory" y "Sayonara Baby", uno para cada sesión de dos horas. Los contenidos de los juegos se corresponden con dos partes de la materia: Las hormonas vegetales en "The Big Plant Theory" y las etapas de desarrollo de las plantas en "Sayonara Baby".

Inicialmente se divide a los estudiantes de un grupo en tres grupos más pequeños y se les facilita una corta historia dinamizadora. En la historia de "The Big Plant Theory" los protagonistas de la serie "The Big Bang Theory" debaten sobre las razones por las que una planta que un personaje le regala a otro no crece. En "Sayonara Baby" el relato un recién graduado en Biología busca trabajo en un vivero llamado Germinator porque se germinan semillas para obtener las plantas. La secretaria de la empresa le dice al graduado que como no sepa mucho del desarrollo de las plantas, su jefe le dirá iSayonara baby! y no le contratará (En la película Terminator el personaje de Arnold Schwarzenegger dice iSayonara baby! a otro personaje cuando le dispara).

Además del relato, se les proporcionan a los estudiantes las reglas del juego. Cada equipo debe elaborar una lista de frases que deben corresponder a materia de los temas que se han visto en teoría o incluso prácticas. Tras la elaboración de las frases, se hacen rondas de preguntas sobre las mismas (por ejemplo, en "The Big Plant Theory" si son verdaderas 0 falsas), en las que se alterna que equipo hace la pregunta y cual responde. El profesor anota 
los aciertos o fallos e indica los posibles errores en las frases. Se puntúa tanto la elaboración de las preguntas como las respuestas. Las reglas completas de los juegos y de su evaluación son más complejas, pero explicarlas aquí sería demasiado prolijo. Al final de cada sesión se le da a cada miembro del equipo ganador una pegatina (Figura 1).

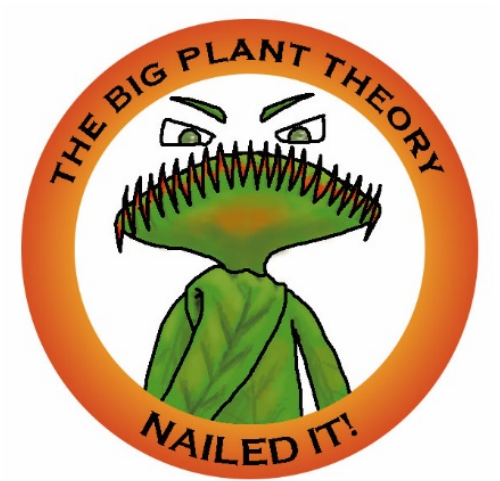

Figura 1. Ejemplo de pegatina usada en grupos reducidos.

\subsection{Plantas transgénicas}

En el Grado de Biología, la Fisiología Vegetal Aplicada es una materia troncal de $3^{\circ}$ de Grado que tiene una carga de 6 créditos ECTS que se reparten en clases magistrales, clases prácticas de laboratorio y docencia en grupos reducidos. En el curso 2011-2012 se comenzó a impartir esta materia con un número de matriculados medio de unos 75 estudiantes. Este número de estudiantes implicaba la existencia de 6-7 grupos reducidos con una media de 12 a 15 estudiantes por grupo con cinco sesiones de dos horas cada una hasta un total de 10 horas.

Las plantas transgénicas es un tema central en la Fisiología Vegetal dada su estrecha relación con la Biotecnología Vegetal sin olvidar sus connotaciones económicas, sociales y éticas. Se eligió este tema por su importancia en el programa de la asignatura y por la posibilidad que ofrecía en la docencia en grupos reducidos, en dónde se podían incorporar nuevas estrategias educativas no desarrolladas hasta el momento en la Fisiología Vegetal Aplicada. Durante las sesiones de grupos reducidos analizábamos los diferentes pasos que nos llevaron desde la 
Revolución del Neolíico a la Revolución Verde. Lo que yo no intuía en ese momento es que la Revolución no sólo sería verde.

Durante los primeros cursos $(2010 / 11,2011 / 12)$ en donde teníamos un número reducido de horas, aplicamos aquellas estrategias bien conocidas, ya que eran las que usábamos habitualmente en las clases magistrales (Moodle, búsquedas en el PubMed, presentaciones orales por parte de los estudiantes en Power Point). Nos inundaba la alegría, la nueva situación de alguna manera era una repetición de la clase magistral pero... un cierto temor comenzó a surgir por sí realmente el trabajo en grupo reducido no era eso.

Pero entonces llegó el curso 2013/14 en dónde se produjo un incremento significativo en el número de horas y de grupos, pasando a repetir las cinco sesiones de un grupo reducido hasta cuatro veces. Esta nueva situación hizo saltar las alarmas. A la alegría le comenzó a acompañar el temor (que había aumentado considerablemente), una cierta furia por no saber cómo enfocar la nueva realidad, desagrado porque realmente no emocionaba lo que se hacía y para terminar, tristeza, aquello no era lo que se esperaba. Teníamos que pensar en alternativas para poder salir de la zona de confort... pero no sólo la nuestra, sino también la de los estudiantes.

En la Universidade da Coruña, desde la implantación del plan Bolonia, contamos con cursos de formación por parte del Cufie que nos proporcionan las bases teóricas y prácticas. Desde el principio nos ha gustado asistir a dichos curso de formación y tenemos que agradecer aquí especialmente a los profesores Francisco Imbermón y Juan Marín, cuyos cursos han sido de lo más motivadores e instructivos. Fue precisamente en uno de esos cursos, a dos días de las sesiones expositivas finales de los cuatro grupos reducidos, cuando comenzamos a escuchar hablar sobre otras estrategias educativas (algunas de ellas ya conocidas y utilizadas en otras ramas del saber Medicina, Enfermería, Derecho...) cómo el estudio del caso, tormentas de ideas, juegos de rol, etc... pero que hasta el momento no se usaban en los estudios de Biología.

Les planteamos a nuestros estudiantes la posibilidad de presentar sus trabajos como si fuera un juego de rol, a modo de programa divulgativo científico en dónde aparecieran los diferentes 
actores relacionados con el mundo de las plantas transgénicas. El resultado fue impresionante. Los estudiantes de los cuatro grupos se pusieron de acuerdo entre ellos 24 horas antes y el día de la exposición aparecieron en clase presentadoras de televisión, científicos eminentes, abogados, personal de laboratorio, agricultores, empresarios, activistas de Greenpeace (Figura 2).
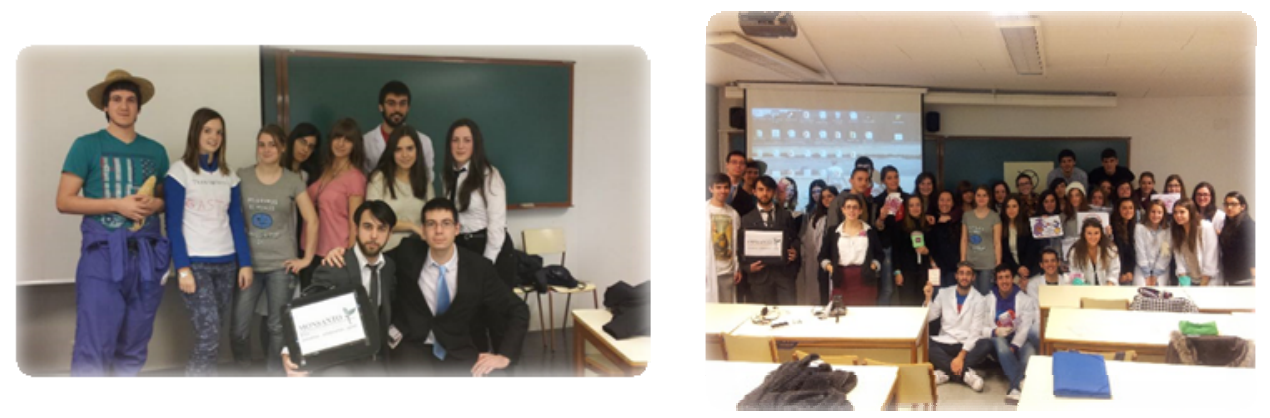

Figura 2. Juego de rol en Curso 2013/14

Decir que el resultado fue bueno sería quedarnos cortos porque la implicación de los estudiantes fue total, expusieron cada uno la parte correspondiente al trabajo de una manera desenfadada y asumiendo su papel recién adquirido. A partir de este momento el juego de rol se incorporó a nuestras sesiones de grupos reducidos, pero era necesario buscar otras alternativas.

Aparecieron los videos. Los estudiantes debían de elaborar un vídeo de 5-8 minutos en dónde quedaran reflejados los diferentes aspectos del tema a tratar. Lo importante en esta actividad es el ingenio y la libertad que tienen los estudiantes en interpretar el conocimiento científico. Se puede utilizar cualquier tipo de formato: reportaje periodístico, publicidad, informativos, juegos, etc... Como resultado, en los años siguientes nos encontramos con videos sobre programas habituales en la TV como "Cuarto Milenio", "Equipo de Investigación", "BBC News", "Viajando con Chester", "Callejeros Viajeros", etc... (Figura 3) 


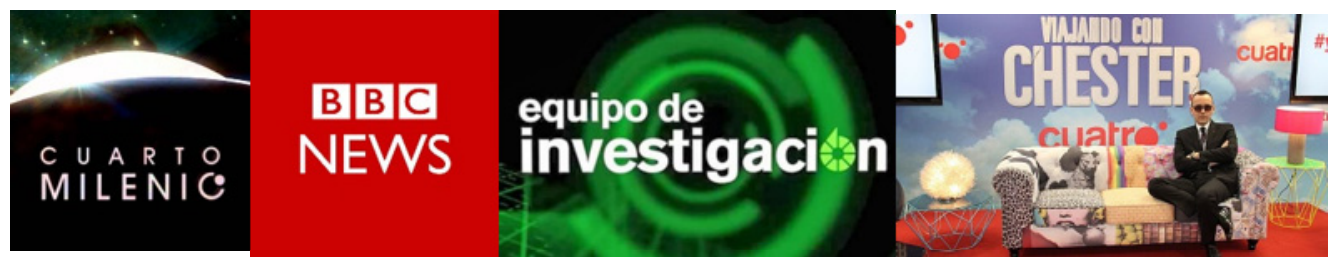

\section{viajêress}

Figura 3. Ejemplos de videos Cursos 2014.15 y 15.16

Pasamos a elaborar posters científicos divulgativos. Los miembros del grupo reducido se dividen en subgrupos de dos personas. Cada subgrupo debe de preparar una presentación tipo poster divulgativo sobre el sujeto de estudio para presentarla al resto de los compañeros en la siguiente sesión de grupo reducido a modo de sesión de paneles como en un congreso convencional. Los estudiantes se irán rotando en el aula (Figura 4) de manera que mientras un estudiante explica su poster a los compañeros, su compañero atiende a la explicación de otro poster. De esta manera se establece una interacción entre los estudiantes en dónde explican y reciben explicaciones.
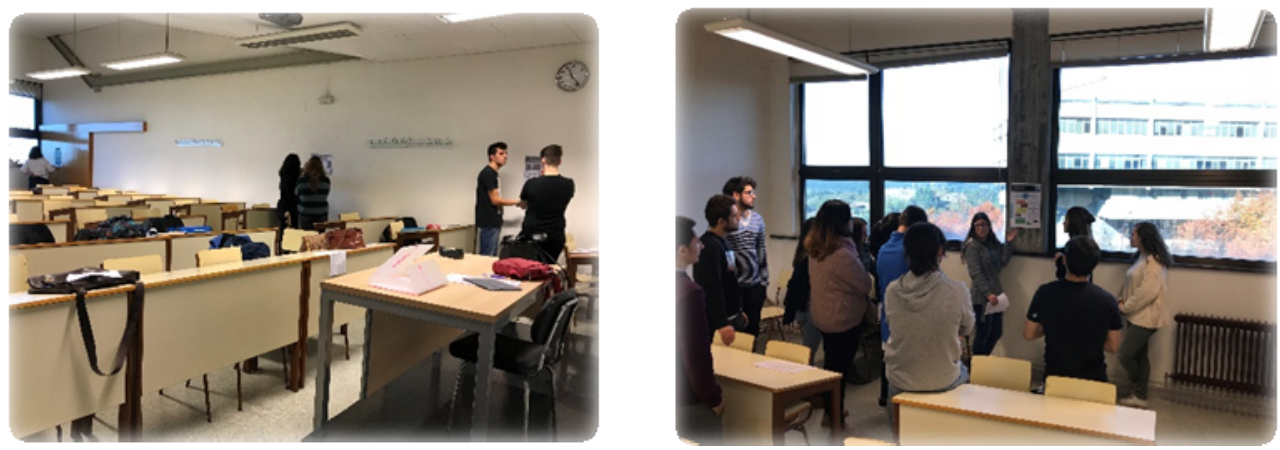

Figura 4. Posters científicos Curso 2017-2018 
Y por último abrimos la puerta a los debates (Figura 5) que no dejaban de ser otro juego de rol. En un tema tan polémico como las plantas transgénicas, el debate era algo conocido de antemano, con estudiantes a favor y en contra.

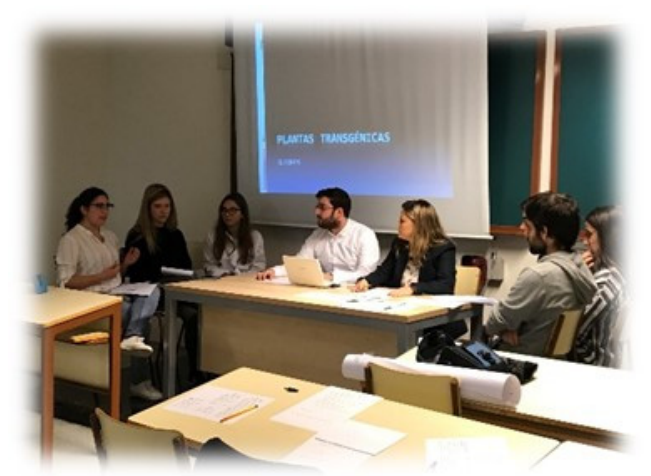

Figura 5. Debate Fin de Sesiones Curso 2016-2017

Fue de esta manera, poco a poco y año tras año, como llegamos en el año 2015-2016 (Figura 6) a nuestro primer congreso sobre Transgénicos, que se realiza todos los años en la última semana de diciembre en la Facultad de Ciencias. Durante el mismo, los estudiantes tienen la opción de realizar presentaciones orales en forma de Power Points, sesión de paneles con sus posters científico-divulgativos, visualización de videos y un debate final moderado por ellos mismos.

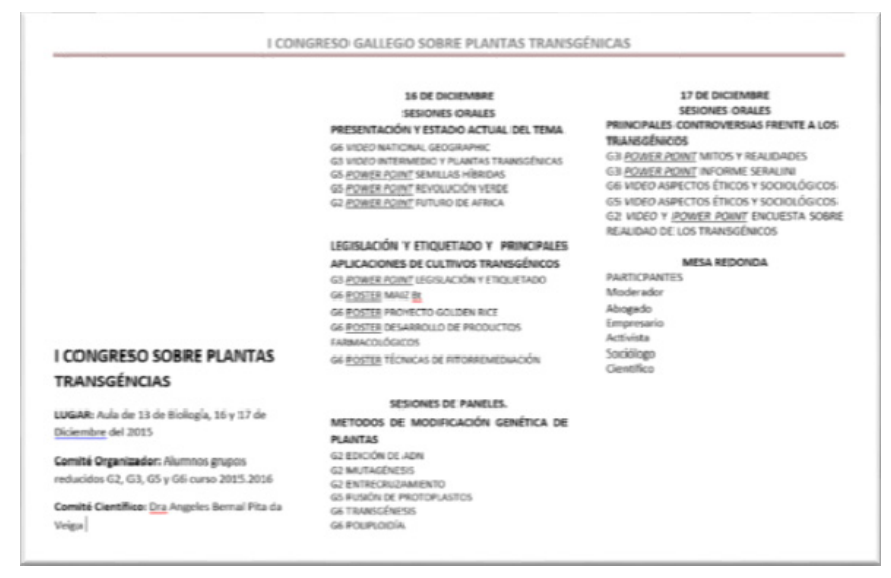

Figura 6. Tríptico I Congreso sobre Transgénicos 
Tenemos también una página en Facebook (FVA2017.2018) cuya denominación va cambiando de curso en curso, y en dónde en el seno de un grupo cerrado, los integrantes del grupo reducido de los diferentes años pueden acceder a información actualizada procedente de publicaciones científicas, fundaciones, sociedades biotecnológicas, prensa nacional e internacional, etc...

\section{RESULTADOS}

\subsection{The Big Plant Theory \& Sayonara Baby}

¿Cómo se llegó a esta estrategia? En cursos anteriores se realizaban otros juegos algo más sencillos que se basaban en hacerles preguntas a los estudiantes, preguntas que elaboraba el profesor. Los problemas que observamos fueron en primer lugar que la metodología era en gran medida pasiva y poco motivadora, y en segundo, los estudiantes se pasaban de curso a curso las preguntas con lo que apenas había espacio para la creatividad y la reflexión.

Los estudiantes valoraron positivamente la experiencia y su actitud ante la asignatura mejoró. Los relatos dinamizadores contribuyeron a ello, y que ellos elaboraran las preguntas les hizo participar más activamente. Curiosamente, algo que parece tan banal como premiarles con una pegatina, fue muy bien recibido y una motivación más.

Las notas de los grupos reducidos mejoraron respecto al curso anterior (de una media de 8,2 a 9,0). Las notas finales de la materia mejoraron: En el curso 2015-2016 superaron la materia un 56,5\% de los estudiantes, mientras que en el 2016-2017 fueron un 64,7\%. Además, en el 2015-2016 obtuvieron calificación de Notable 0 superior un 8\% de los estudiantes, mientras que en el 2016-2017 subió a un 18\%. En comparación con los resultados de la asignatura equivalente en licenciatura (Pomar et al., 2012) también se constató una mejoría. De todas formas, también hubo algunos cambios en las clases magistrales y los exámenes que pudieron influir en los resultados finales.

Finalmente, esta experiencia sirvió para detectar y corregir errores de concepto de los estudiantes. Esto fue un complemento excelente de las clases magistrales y de las de laboratorio. 
No obstante, como los estudiantes del presente curso 2017-2018 pueden ser alertados por los del 2016-2017 de las reglas de estos juegos, nos planteamos la posibilidad de cambiarlos el presente curso por otros de naturaleza semejante y así mantener el factor sorpresa. Dicho factor nos parece esencial, y se consiguió dentro del mismo curso 2016-2017 con algunos aspectos de las reglas.

\subsection{Plantas transgénicas}

El trabajo que aquí se presenta es el resultado de una experiencia en dónde ha habido grandes aciertos y también errores. Es importante señalar que muchas veces la implementación de estas nuevas estrategias se convierte en una apuesta arriesgada, que se escapa del perfil del docente clásico no siendo adecuadamente valorada por los otros docentes e incluso a veces, por los propios estudiantes que no están acostumbrados a estas innovaciones Uno de los puntos fuertes de esta experiencia es la alta implicación de los estudiantes, la gran flexibilidad que aporta el grupo reducido, la tasa de éxito, el desarrollo de competencias transversales y la promoción de la divulgación. Y como aspectos a mejorar, el sistema de evaluación (es necesario desarrollar rúbricas para cada una de las actividades), los estudiantes en ocasiones se quejan de un exceso de trabajo, la necesidad de explorar nuevas estrategias y la incorporación de nuevas TICs.

\section{CONCLUSIONES}

En las dos experiencias descritas en este trabajo se han obtenido resultados positivos tanto en motivación como en desarrollo de un pensamiento crítico, y ha servido para poner de relieve entre los estudiantes el valor del trabajo en grupo y de los conocimientos del campo de la Fisiología Vegetal. Para terminar, queremos recordar una cita de uno de los actuales gurús del aprendizaje, Sugata Mitra: "No existe aprendizaje sin emoción”. 


\section{REFERENCIAS}

- Pomar, F., Bernal, A., Díaz, J., Silvar, C., Gutiérrez, J. J., Veloso, J., Novo, E. \& Merino, F. (2012). Fisiología vegetal: de la licenciatura al grado en la Universidade da Coruña. En: P. Membiela, N. Casado y M. I. Cebreiros (Ed.) El Espacio Europeo de Educación Superior y la educación por competencias pp 401-404. Ourense. Educación Editora.

- Susanne, C. (2003). Biology before and after Bologna. Bioscience Education Ejournal, 2 (1), pp. 1-9. DOl: 10.3108/beej.2003.02000008. 